\title{
Gradhiva
}

GRADHIV

Revue d'anthropologie et d'histoire des arts

\section{Woodstock en Amazonie et la superstar du ghetto de Kingston}

Les droits patrimoniaux et le droit moral face aux réalités du terrain Woodstock in Amazonia and the Kingston Ghetto Superstar. Patrimonial and moral rights confronted with reality in the field

\section{Laurent Aubert}

\section{OpenEdition}

\section{Journals}

Édition électronique

URL : http://journals.openedition.org/gradhiva/1889

DOI : $10.4000 /$ gradhiva. 1889

ISSN : $1760-849 x$

\section{Éditeur}

Musée du quai Branly Jacques Chirac

\section{Édition imprimée}

Date de publication : 24 novembre 2010

Pagination : 140-157

ISBN : 978-2-35744-029-6

ISSN : 0764-8928

Référence électronique

Laurent Aubert, «Woodstock en Amazonie et la superstar du ghetto de Kingston », Gradhiva [En ligne], 12 | 2010, mis en ligne le 24 novembre 2013, consulté le 03 mai 2019. URL : http:// journals.openedition.org/gradhiva/1889; DOI : 10.4000/gradhiva.1889 

Laurent Aubert

Fig. 1 Enfants jouant avec des CD au Vietnam (détail), 2002. Photo Philip Jones Griffiths (c) Philip Jones Griffiths/ Magnum Photos.

- $\bullet$

1. L'album Deep Forest, publié en 1992, avait en son temps suscité certaines réactions : vendu à plus de 2,5 millions d'exemplaires, il incluait des échantillons remixés de musiques enregistrées chez les Pygmées BaBenzélé de Centrafrique ou les 'Aré 'aré des îles Salomon, utilisés sans l'autorisation ni des ethnomusicologues les ayant enregistrées - respectivement Simha Arom et Hugo Zemp - ni a fortiori des interprètes de ces musiques (voir Aubert 2001 : 113-114).

\section{Woodstock en Amazonie et la superstar du ghetto de Kingston Les droits patrimoniaux et
le droit moral face aux réalités du terrain Les droits patrimoniaux et
le droit moral face aux réalités du terrain}

Prologue

Un domaine dans lequel les questions de copyright se posent de façon particulièrement significative est celui de l'édition musicale. En effet, contrairement à une publication écrite ou audiovisuelle, qui est signée, et donc explicitement assumée par son auteur, le $\mathrm{CD}$ - ou tout autre support audio - donne à entendre la musique en elle-même, extraite de son contexte, et à la fois réifiée et idéalisée par les effets de la prise de son, de la sélection musicale, du montage et de la production.

Pour la plupart des disques publiés dans des collections de type ethnomusicologique, le CD fait aussi entrer la musique dans un circuit économique échappant totalement au contrôle - et parfois même à la connaissance - des individus et des groupes dont elle provient. Quelle garantie ces derniers peuvent-ils avoir qu'on n'abuse pas de leur patrimoine musical et que personne n'est en train de se faire de l'argent sur leur dos ? L' « affaire Deep Forest ${ }^{1}$ » (Feld 1996, Zemp 1996) et quelques autres exemples retentissants ont démontré que cela pouvait arriver. Mais, dans l'immense majorité des cas, le problème provient essentiellement des malentendus suscités par la distance culturelle séparant les musiciens enregistrés - « l'artiste » ou «l'interprète ", selon le jargon de la production musicale - et le marché du disque auquel est destiné le CD. Il est alors de la responsabilité du collecteur d'expliquer le sens de sa démarche aux musiciens avec lesquels il travaille, ainsi que de détailler les garanties et les éventuels avantages que peuvent offrir la publication et la diffusion d'une musique hors de ses frontières culturelles ordinaires.

Les questions relatives à la "propriété intellectuelle», à la «diversité culturelle » et à la sauvegarde du " patrimoine culturel immatériel de l'humanité » ont récemment faitl'objet de plusieurs déclarations officielles édictées par les diverses 
organisations internationales dédiées au domaine culturel (Unesco, OMPI, ICTM², etc.). Ces textes normatifs mettent un accent tout particulier sur les «meilleures pratiques " (best practice) que les anthropologues, les ethnomusicologues et leurs partenaires se doivent de respecter dans les différentes phases de leur travail.

En ce qui concerne le droit d'auteur, l'OMPI stipule par exemple, dans sa section consacrée à la "Gestion collective du droit d'auteur et des droits connexes", qu'il « comprend deux grandes catégories de droits : les droits patrimoniaux et le droit moral» :

Les droits patrimoniaux sont les droits de reproduction, de radiodiffusion, d'interprétation ou d'exécution publique, d'adaptation, de traduction, de récitation publique, de représentation publique, de distribution, etc. Le droit moral comprend le droit de l'auteur de s'opposer à toute déformation, mutilation ou autre modification de son œuvre qui risquent d'être préjudiciables à son honneur ou à sa réputation.

Les deux catégories de droits appartiennent au créateur, qui peut les exercer librement. Par exercice des droits, il faut entendre le droit du créateur d'utiliser l'œuvre luimême, d'autoriser quelqu'un d'autre à l'utiliser ou d'en interdire l'utilisation par des tiers. Le principe fondamental qui sous-tend le droit d'auteur est que les œuvres protégées ne peuvent pas être utilisées sans l'autorisation du titulaire de droits. Néanmoins, certaines législations nationales prévoient des exceptions limitées à cette règle générale. En principe, la durée de protection s'étend à la vie de l'auteur plus un minimum de cinquante années après sa mort.

Ces aspects juridiques sont stipulés dans des conventions internationales auxquelles la plupart des pays sont actuellement parties. Au moment de leur adhésion, les États membres devraient avoir une législation nationale conforme aux normes internationales 3 .

Quant à l'ICTM, il préconise que, lorsqu'il effectue des enregistrements sur le terrain, le chercheur établisse avec ses interlocuteurs musiquants des protocoles clairs relatifs à l'usage qui pourra être fait de ces documents sonores, ceci afin d'éviter toute équivoque. Ces protocoles devraient déterminer les différents droits attribués à «l'interprète» (l'individu ou le groupe enregistré), ceux qu'il concède au collecteur et aux archives où les enregistrements seront déposés, et enfin ceux qui reviendraient à chacun dans l'éventualité d'une publication, y compris sur Internet. Ces protocoles devraient évidemment aussi indiquer les limites que l'interprète ou sa communauté pourraient éventuellement imposer à cette diffusion. "Ici se posent de véritables questions éthiques, souligne Anthony Seeger. Si l'artiste émet des restrictions à propos de quelque chose, le collecteur ou le contributeur, ainsi que les archives, devraient prendre toutes les mesures possibles pour respecter ces souhaits. On peut y voir un obstacle à la diffusion, mais cela devrait être un principe fondamental de la politique suivie par les archives. » (2007: 74)

Or, plus la distance culturelle entre ceux qui produisent une musique, ceux qui la collectent et, éventuellement, ceux qui la publient est grande, plus les

- $\bullet$

2. OMPI (en anglais WIPO) : Organisation mondiale de la propriété intellectuelle; ICTM : International Council for Traditional Music. Voir notamment à ce propos : Duvignaud et Khaznadar 2004, Pennewaert 200?, Aubert (dir.) 2009, Trottier 2010.

3. http://www.wipo.int/about-ip/fr/ about_collective mngt.html. risques de malentendu sont élevés, surtout en ce qui concerne les questions financières. Comment en effet expliquer, par exemple, aux représentants d'une communauté amérindienne de la forêt amazonienne que la publication d'un CD dédié à leur musique coûtera toujours plus qu'elle ne rapportera? Et corrélativement, comment les convaincre des raisons pour lesquelles une telle publication paraît néanmoins importante à ceux qui la leur proposent? Il est donc nécessaire qu'un réel dialogue autour des enjeux - notamment patrimoniaux - du travail de l'ethnomusicologue s'instaure sur le terrain même afin de créer un climat de confiance et dissiper toute équivoque. 


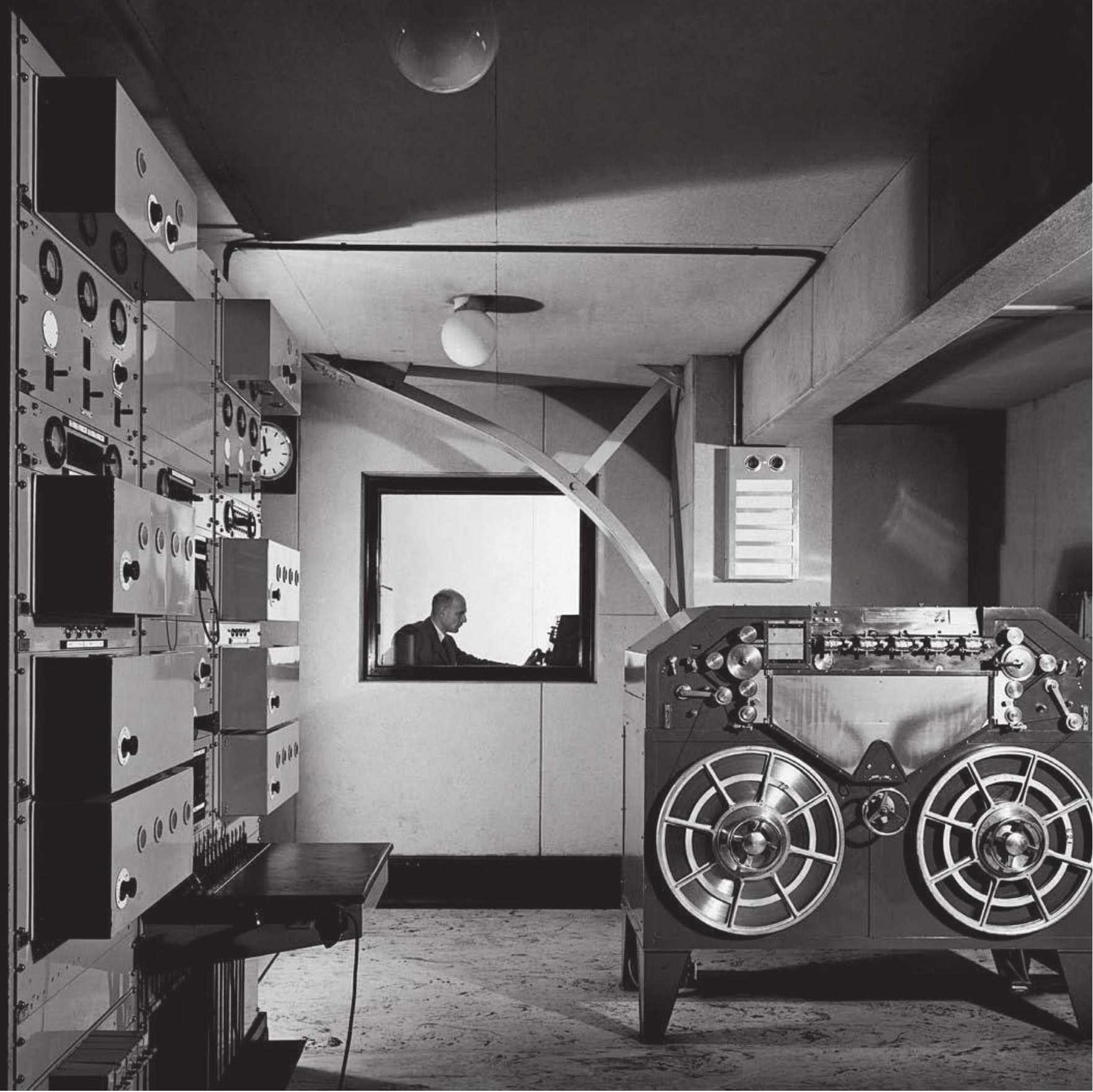

Fig. 2 Enregistrement dans la Tape Room n 2 de la BBC, Londres, 20 octobre 1937 @ BBC/Corbis. 
Une opération de sauvegarde autogérée : le cas awajún-wampis

À ce propos, j'aimerais évoquer un cas auquel j'ai récemment été confronté en tant que responsable scientifique de la collection de CD des AIMP (Archives internationales de musique populaire4), que je dirige dans le cadre de mon travail au musée d'Ethnographie de Genève. Essentiellement dédiée aux travaux de terrain d'ethnomusicologues, cette collection est publiée par un petit éditeur suisse, VDE-Gallo. Elle comporte aujourd'hui près d'une centaine de titres, dont la majorité provient d'enregistrements recueillis sur place et reproduits "en l'état ", c'est-à-dire sans arrangements de studio ni concessions particulières aux lois du marché. L'accent y est mis sur des musiques issues d'anciennes traditions orales, mais dont les répertoires, les particularités esthétiques et les occasions de jeu sont aujourd'hui souvent sujets à de profondes mutations en raison de l'influence des médias modernes et des nouvelles technologies sur les goûts et les pratiques culturelles. Un grand nombre des documents publiés dans la collection AIMP sont ainsi consacrés à des musiques certes belles, significatives et parfois rares, mais vulnérables : ils ont avant tout valeur de traces mémorielles. Le potentiel commercial de cette collection est par conséquent plus que limité; pour être plus précis, elle ne pourrait simplement pas exister sans le soutien financier du musée.

C'est en cette qualité qu'en 2005 j'ai été approché par l'anthropologue canadien Jeremy Narby, qui travaillait en Amazonie péruvienne depuis de nombreuses années. Souhaitant réaliser un $\mathrm{CD}$ consacré aux chants et à la musique de deux communautés amérindiennes de cette région, les Awajún et les Wampis de la vallée du Cenepa, il estimait que les AIMP étaient le partenaire le plus approprié pour faire aboutir ce projet. Celui-ci s'inscrivait pour lui dans le cadre d'une action humanitaire menée au sein de l'ONG Nouvelle Planètes, présente dans plus de vingt pays d'Afrique, d'Asie et d'Amérique du Sud. Un des principes de Nouvelle Planète, m'a-t-il précisé, était de développer sur le terrain des collaborations avec des associations représentatives des communautés concernées. En l'occurrence, le partenaire de l'ONG en Amazonie péruvienne était l'organisation autochtone Odecofroc (Organización de Desarrollo de las Comunidades Fronterizas del Cenepa: Organisation de développement des communautés frontalières de la rivière Cenepa), désignée par les cinquante-trois communautés amazoniennes vivant dans le bassin du Cenepa, au Pérou, pour les représenter vis-à-vis de l'extérieur ${ }^{6}$.

L'idée d'enregistrer les chants des Awajún et des Wampis, me dit Narby, émanait des Indiens eux-mêmes, qui pressentaient les risques d'oubli et de fragmentation de leur patrimoine culturel. Connaissant bien les motivations de l'anthropologue canadien, les représentants de l'Odecofroc avaient souhaité organiser avec lui une campagne systématique de collecte des chants et de la musique de leur communauté afin d'en préserver la mémoire et de contribuer à sa pérennisation. Une première série d'enregistrements avaient déjà été réalisée en 2004 par le musicien suisse Franz Treichler, collaborateur de Narby, et une autre était prévue pour 2006.

Il est intéressant de noter que cette campagne a été en grande partie financée par les recettes d'un spectacle monté par les Young Gods, le groupe de rock électronique fondé par Treichler, avec la collaboration spéciale de Narby, sous le titre Amazonia Ambient Project. «Pour l'occasion, nous informe le site Internet du groupe, les Young Gods seront en petite formation électronique (ordinateur, échantillonneur et percussions) et ils mêleront la présentation de leur dernier album ambient, Music for Artificial Clouds (2004), avec des plages sonores enre- 


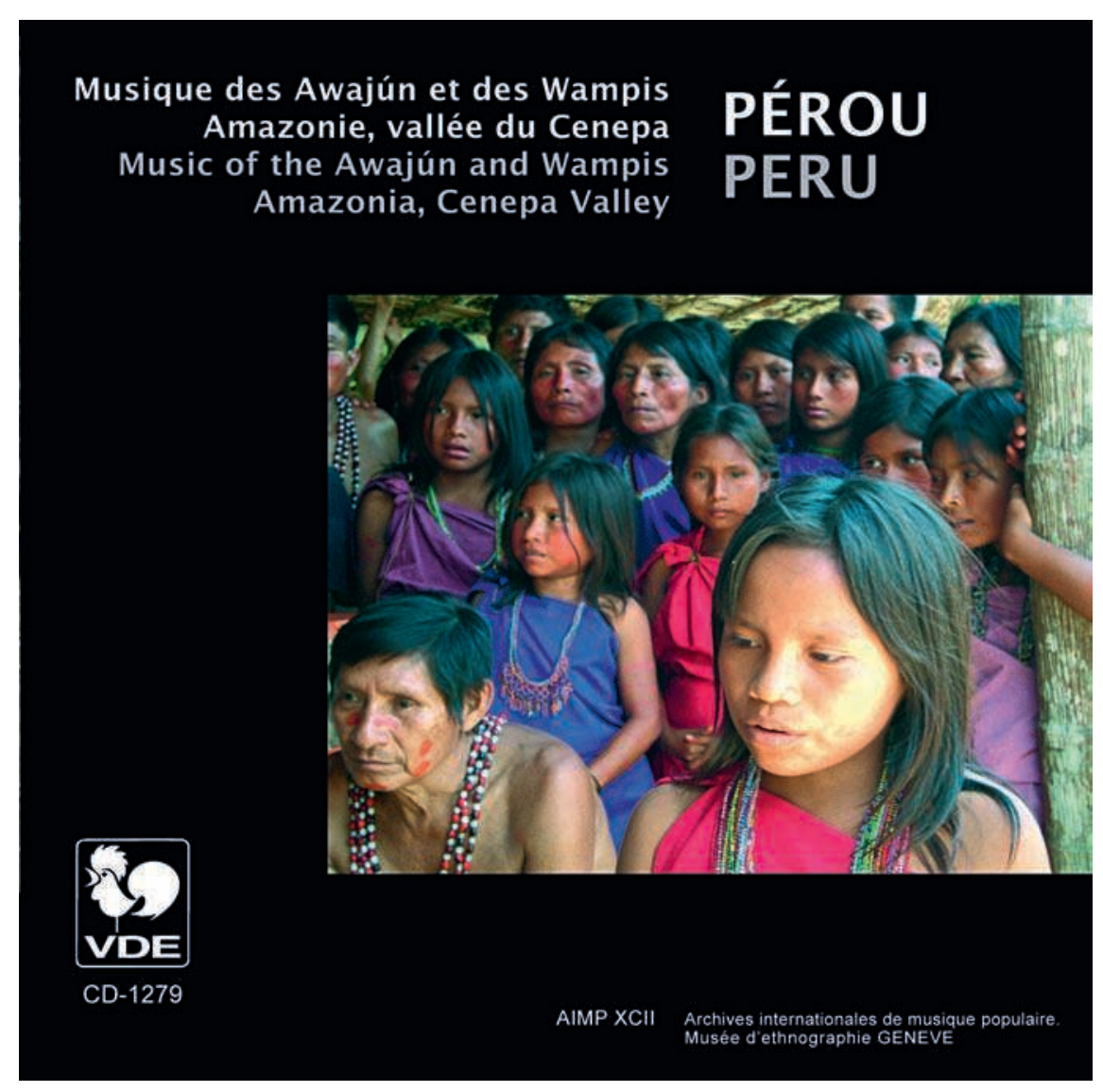

Fig. 3 Couverture du disque Musique des Awajún et des Wampis, Amazonie, vallée du Cenepa, Pérou, enregistrements de Franz Treichler; textes de Raúl Riol et Jeremy Narby. Éd. VDE-1279.

gistrées en Amazonie et retravaillées par ordinateur : une œuvre d'improvisation électronique?. " Il faut préciser que, contrairement à Deep Forest, celle-ci n'incluait aucun enregistrement de musique amérindienne, mais uniquement des «ambiances naturelles» captées dans la forêt amazonienne : l'éthique du copyright était donc respectée puisque aucun patrimoine humain n'y était mis à contribution ${ }^{8}$.

Ce programme qui, selon le journal Le Courrier, « incarnait une forme particulière d'engagement artistique en faveur de la biodiversité et la défense des peuples indigènes [...] », permit, comme l'affirmait Treichler, «de financer en partie un projet d'archivage de musique jivaro du nord de l'Amazonie, en collaboration avec l'ONG Nouvelle Planète et le musée d'Ethnographie de Genève. Le résultat sera publié l'an prochain par les Archives internationales de musique populaire du musée. Ce projet rejoint complètement notre motivation profonde en musique, d'ailleurs peut-être un peu utopique : développer un langage qui ait un impact positif sur notre environnement ${ }^{9} »$.

Sur place, ce projet prit la forme d'un «premier festival de musique awajún du Cenepa» (Nagkamsa Awajún nampet dakubau atatui Cenepia; Dasen 2009), organisé sur trois jours par et pour les Awajún eux-mêmes, en présence de la communauté entière et avec un minimum d'intervention externe. L'événement fut filmé ${ }^{10}$ et enregistré par l'équipe de l'anthropologue. Il permit de réunir plus
Plusieurs extraits musicaux de cet album sont consultables sur http://gradhiva.revues.org/1813

\section{- \\ 7. http://www.younggods.com/ $\mathrm{cms} /$ front content.php?client $=1 \& \mathrm{l}$ ang $=18$ cidcat $=248$ cidart $=33$.}

8. Si Music for Artificial Clouds des Young Gods ne comporte effectivement pas de musique amérindienne, en revanche l'Amazonia Ambient Project - jamais publié - incluait des extraits remixés de chants du chamane ashaninka Juan Flores Salazar, lequel avait été largement rémunéré pour différentes collaborations aux travaux de Jeremy Narby (voir Huxley et Narby 2002 : 318-325; Narby 2005 : 33-41; www.mayantuyacu.com]

9. «Les Young Gods accèdent au "son chamanique" », interview de Franz Treichler par Roderic Mounir, Le Courrier, Genève, 25 octobre 2008.

10. Un film a d'ailleurs été produit et réalisé sur ce « festival » sous le titre Woodstock in the Amazon [2007) par le vidéaste étasunien John Hazard, qui faisait partie de l'équipe de Jeremy Narby. 
d'une centaine de pièces vocales et instrumentales, interprétées en solo ou en duo par soixante-quatorze personnes différentes, hommes et femmes, tant awajún que wampis. Les paroles des chants ont ensuite été transcrites et traduites, et la musique partiellement analysée.

Comme ce matériel musical était trop important pour figurer intégralement sur un $C D$, il a été résolu d'en faire une sélection représentative d'un peu plus d'une heure, quitte à ce que l'intégrale en soit un jour rendue accessible sur Internet. Or cette sélection excluait de fait plus de la moitié des interprètes. Pour ne froisser personne, nous avons donc proposé à Zebelio Kayap, président de l'Odecofroc, que ce soient les villageois qui en opèrent le choix. La réponse ne se fit pas attendre: "Comment voulez-vous que nous choisissions? Chaque personne a son chant, et chaque chant est beau : ce serait la guerre entre nous!» Comprenant néanmoins les impératifs de la production discographique, ils nous ont donc demandé d'effectuer la sélection nous-mêmes, ce que nous avons fait en nous efforçant d'adopter des critères à la fois esthétiques, typologiques et anthropologiques :

- esthétiques par la diversité des timbres et des formes mélodiques, par l'équilibre entre voix d'hommes et de femmes ainsi qu'entre pièces vocales et instrumentales;

- typologiques par une répartition équilibrée entre pièces rituelles (anen) et profanes (nampeg), et aussi entre les diverses thématiques abordées dans les paroles des chants (Riol et Narby 2009: 5-9);

- et enfin anthropologiques en proposant une représentation aussi proportionnelle que possible des différentes collectivités villageoises engagées dans le processus.

\section{L'impossible contrat, ou lorsque deux logiques s'affrontent}

Une fois cette sélection faite et approuvée par la communauté - une copie en avait été envoyée à l'Odecofroc -, il s'est agi de préparer l'accord écrit qui nous liait pour la production de ce CD. J'ai donc proposé à Narby le «contrat-type » des AIMP, que nous, en tant que "producteur", signons généralement avec l'ethnomusicologue ou le preneur de son, lequel y apparaît comme «auteur» (sousentendu des enregistrements et des textes du livret). Or il est rapidement apparu que ce contrat-type ne pouvait pas convenir tel quel à ce projet. Ce qui n'était pour moi qu'une formalité posait en fait de réels problèmes moraux et déontologiques à Narby, en particulier les clauses relatives à la question du droit d'auteur sur les enregistrements et à celle de l'identification des ayants droit.

En effet, le contrat stipulait notamment que «l'auteur cède [...] au producteur le droit d'édition exclusif, illimité et transmissible des enregistrements [...] ", et donc qu'il «se porte garant [...] de l'intégralité de ses droits de cession au moment de la conclusion du [...] contrat ». Cette formulation commode a évidemment comme principal objet de dédouaner l'éditeur en cas de litige avec un tiers et de reporter la responsabilité légale sur les épaules du chercheur, qui est de fait souvent le seul à pouvoir traiter en confiance avec ses informateurs. Mais notre anthropologue estimait n'avoir aucun droit sur ces enregistrements, et surtout pas celui de figurer sur le contrat comme « auteur » de ce projet. Il se situait tout au plus en tant que "facilitateur" et contributeur pour ce qui était d'une partie des textes prévus pour le livret. L'auteur ne pouvait à son sens être que les communautés

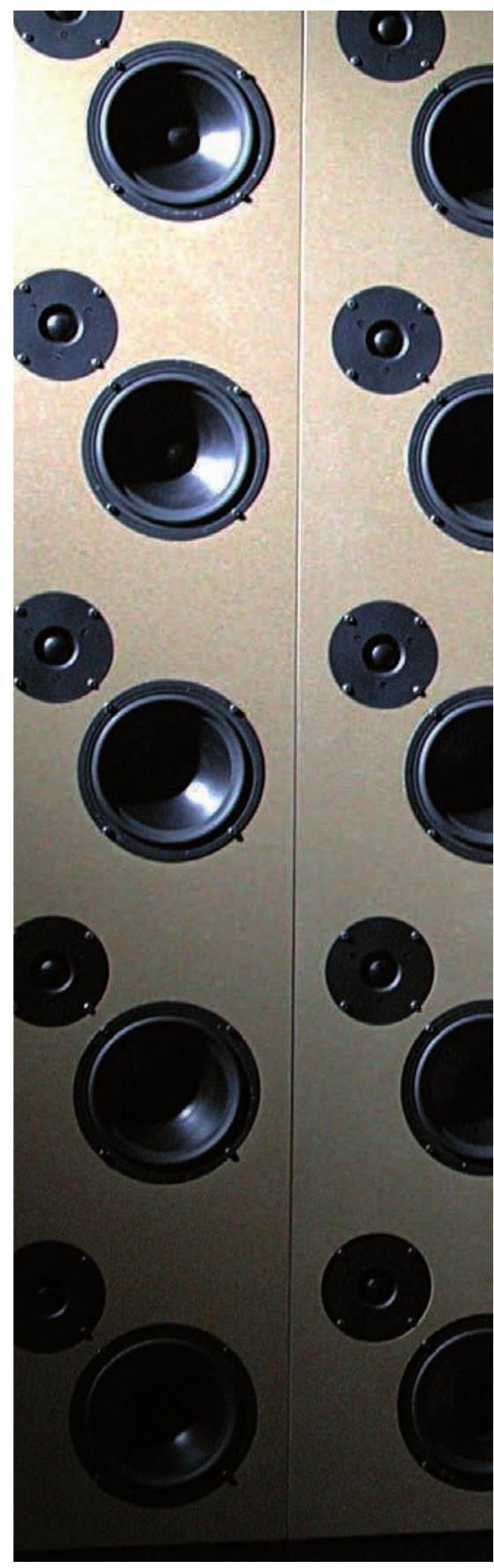




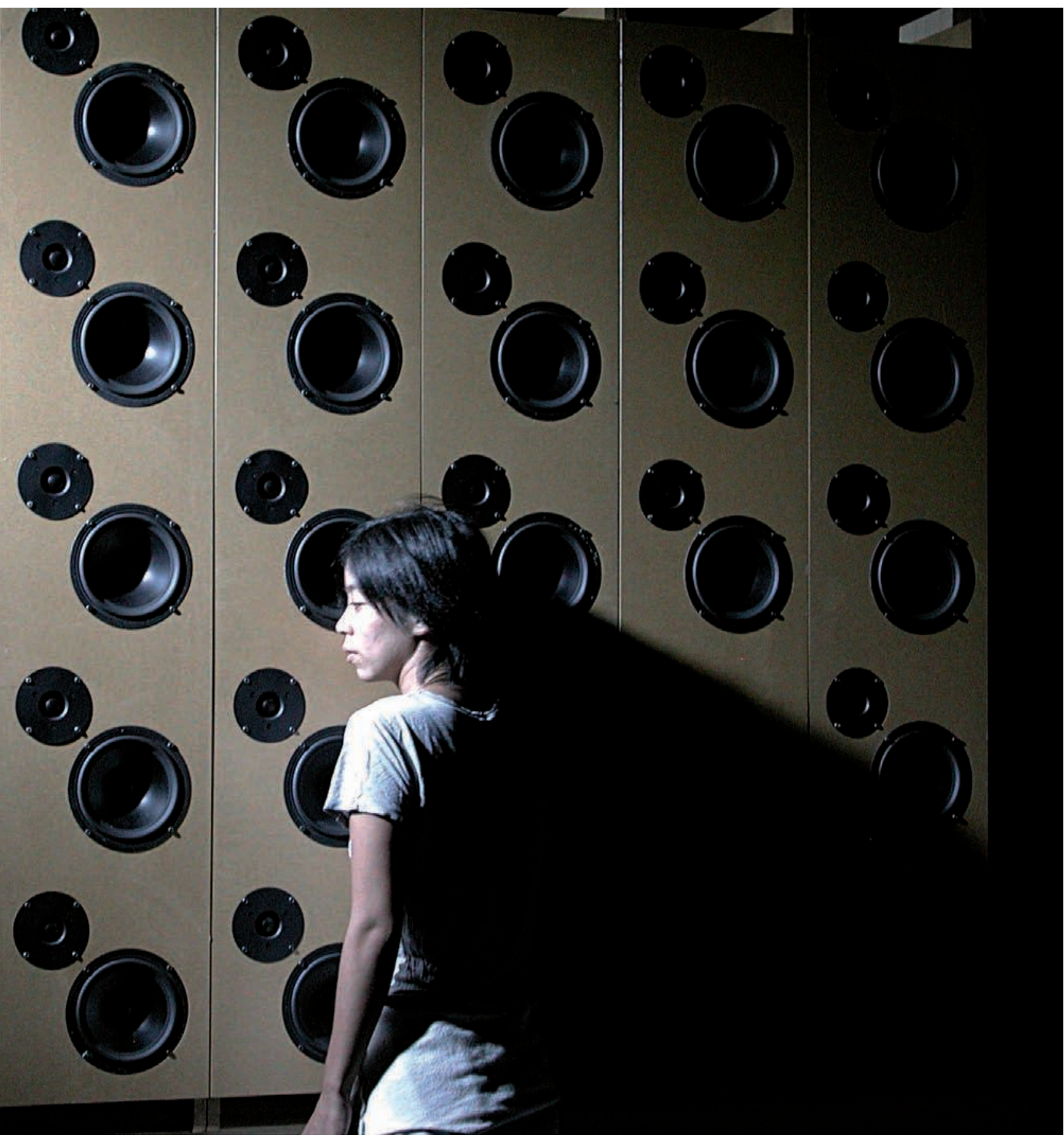

Fig. 4 Edwin van der Heide, A World Beyond the Loudspeaker, exposé à la BIAS Sound Art Exhibition, Taipei Fine Arts Museum, 2005. Avec l'aimable autorisation du Studio Edwin van der Heide. 
awajún et wampis elles-mêmes, détentrices tant des «droits patrimoniaux » que du «droit moral» sur la musique. Et comme l'organisme qu'elles avaient démocratiquement désigné pour défendre leurs intérêts vis-à-vis du monde extérieur était l'Odecofroc, c'était donc, en toute logique, au président de cette dernière, Kayap, qu'il revenait de signer le contrat qui devait nous lier'1.

Il est ainsi apparu important que le contrat distingue clairement ce que l'OMPI et d'autres organisations internationales désignent du terme de " propriété intellectuelle ", qui, dans le domaine du patrimoine culturel immatériel, se rapporte à une œuvre, un corpus ou un répertoire particulier, et les droits d'édition - exclusifs ou non-, qui ne concernent que les enregistrements réalisés à partir d'éléments de ce patrimoine. Et cette distinction n'était pas évidente à saisir pour les Awajún et les Wampis. En outre, cette expression de "propriété intellectuelle» ne correspondait pas à la réalité des faits dans leur culture : pour eux, chaque chant est un trésor personnel, jalousement gardé et transmis soit par un parent proche de même sexe, soit par un esprit lors d'un rêve ou d'une transe induite par l'ingestion de plantes hallucinogènes en situation rituelle. Mais l'individu détenteur d'un chant n'en est pas pour autant le propriétaire; il n'en est que le dépositaire, dans la mesure où il l'a acquis de façon régulière, et ce dépôt n'est en tout cas ni collectif, ni négociable, ni transmissible hors de ce cadre. Pour lever toute ambiguïté, il nous a donc fallu rajouter une clause au contrat stipulant que : «Les droits cédés par L'AUTEUR au PRODUCTEUR ne concernent que les enregistrements mentionnés, et pas les morceaux eux-mêmes, qui demeurent partie intégrante du patrimoine culturel du peuple awajún.»

La question financière est aussi évidemment centrale dans ce genre de collaboration, et elle peut générer un certain nombre de tensions et de malentendus. En effet, comment expliquer à des gens vivant en pleine forêt amazonienne, dans un dénuement presque total, que non seulement personne ne gagnera d'argent sur cette publication, mais qu'elle ne pourrait simplement pas être réalisée sans un apport financier à fonds perdus, en l'occurrence de l'ordre de 5000 à 6000 euros? Une telle somme était évidemment faramineuse pour les Awajún et les Wampis, qui vivent encore largement en marge de la société de consommation - surtout si l'on considère qu'elle venait s'ajouter au financement de la campagne d'enregistrement, lui-même généré par les bénéfices de l'Amazonia Ambient Project mentionné plus haut. Même si les frais de production d'un tel CD sont presque insignifiants comparés à ceux en vigueur dans la variété internationale et la majorité des musiques "actuelles", les montants en jeu dans cette opération avaient néanmoins quelque chose de totalement incongru en regard des conditions d'existence de nos partenaires amazoniens. Il fallut donc leur expliquer que, provenant de fonds spécifiques, cet argent n'aurait de toute façon pas pu leur être attribué à d'autres fins.

S'est alors posée la question des royalties. Rappelons à ce sujet que, selon l'usage dans les milieux de la production musicale, les royalties d'un CD ne sont versées à «l'artiste " qu'une fois les frais de production couverts par les bénéfices des ventes. Or, d'après nos calculs réalisés à partir de disques de notre collection au potentiel comparable, il aurait fallu attendre plus de soixante-dix ans avant que les Awajún aient la chance de toucher le moindre centime si nous appliquions rigoureusement ce principe au projet qui nous liait. Et encore faudrait-il que le disque reste aussi longtemps disponible sur le marché et qu'il génère des ventes régulières sur une aussi longue période - ce qui n'est jamais arrivé. Or il est évident que l'évolution des technologies ne permet pas d'imaginer l'avenir du 
marché du disque, qui est d'ailleurs en train de s'effondrer. Quant aux ventes par Internet, il paraît difficile de supposer qu'elles deviennent un jour rentables pour une musique de cette nature.

Pour cette raison, nous avons adopté pour ce type de production le principe d'un montant forfaitaire de 2500 francs suisses (environ 1800 euros), versé à «l'auteur» à la signature du contrat. Cette somme correspond concrètement à l'achat des droits d'utilisation exclusive des enregistrements sélectionnés ainsi que des textes et des photographies qui les accompagnent. L'auteur, quel qu'il soit, assume donc être en possession de ces droits, et il lui appartient ensuite de gérer et, éventuellement, de répartir la somme à sa guise. Vingt-cinq exemplaires du CD lui sont par ailleurs remis gratuitement, et il peut en outre acquérir autant d'exemplaires supplémentaires qu'il le souhaite au "prix d'auteur", qui correspond à la moitié du prix de vente au public en Europe occidentale, soit 10 euros (le coût moyen d'un tel CD sur le marché étant d'environ 20 euros). Le cas échéant, et pour autant que l'auteur en ait la possibilité, cette clause lui permet de réaliser ainsi quelques gains supplémentaires par les ventes directes du $\mathrm{CD}$, sur lesquelles il peut effectivement toucher un bénéfice de l'ordre de $50 \%$. Le problème est évidemment que ces tarifs sont totalement exorbitants pour le marché péruvien, et plus encore pour les Awajún et les Wampis; sans parler des frais d'envoi et de douane qui viendraient s'y ajouter. La solution que nous avons donc proposée à l'Odecofroc a été de leur octroyer dans notre contrat un droit de licence particulier et non exclusif, couvrant l'ensemble du continent sud-américain, et ceci pour une durée illimitée. Cette clause a été d'autant plus facile à intégrer que la maison VDE-Gallo n'a actuellement pas de distributeur dans cette région du monde.

Une autre éventuelle source de revenus qui pourrait survenir - encore que cette occurrence soit rarissime, totalement imprévisible et sujette à débats serait celle qui pourrait provenir des "droits voisins " - ou "droits annexes "générés par l'acquisition par un tiers des droits d'utilisation de tout ou partie d'une production discographique à des fins commerciales. À cet égard, le point VII du «contrat-type» des AIMP, «Droits annexes", stipule qu'« en cas de cession à des tiers pour une autre utilisation que celle prévue dans le présent contrat, LE PRODUCTEUR versera à L'AUTEUR une redevance égale à $50 \%$ des bénéfices qu'il aura effectués de la sorte ". Or, depuis le début de la collaboration entre les AIMP et VDE-Gallo en 1984, il est parfois arrivé que des organismes à but non lucratif demandent l'autorisation d'utiliser des extraits de documents publiés - et donc soumis au copyright - dans le cadre de projets de nature associative et/ou caritative; chacune de ces requêtes a été examinée et, selon les cas, acceptée ou rejetée d'un commun accord entre l'éditeur, le responsable de la collection et les détenteurs des droits d'auteur de la production concernée. Mais - du moins jusqu'à très récemment - aucune requête chiffrée ne nous a jamais été adressée pour l'acquisition de droits sur un disque de la collection AIMP.

La loi du plus fort...

Les questions soulevées par cet exemple amazonien et les négociations qu'il a suscitées touchent ainsi à des aspects essentiels de la déontologie professionnelle mise au service de la protection des droits patrimoniaux. Elles s'inscrivent en outre dans la perspective de l'« ethnomusicologie d'urgence », depuis longtemps 
Fig. 6 Christian Marclay, vue de l'installation Echo and Narcissus, 1992/1999, Tokyo Opera

City Gallery, Tokyo, 1999.

$15000 \mathrm{CD}$ de dimensions

variables (c) Christian Marclay.

Avec l'aimable autorisation de la

Paula Cooper Gallery, New York.

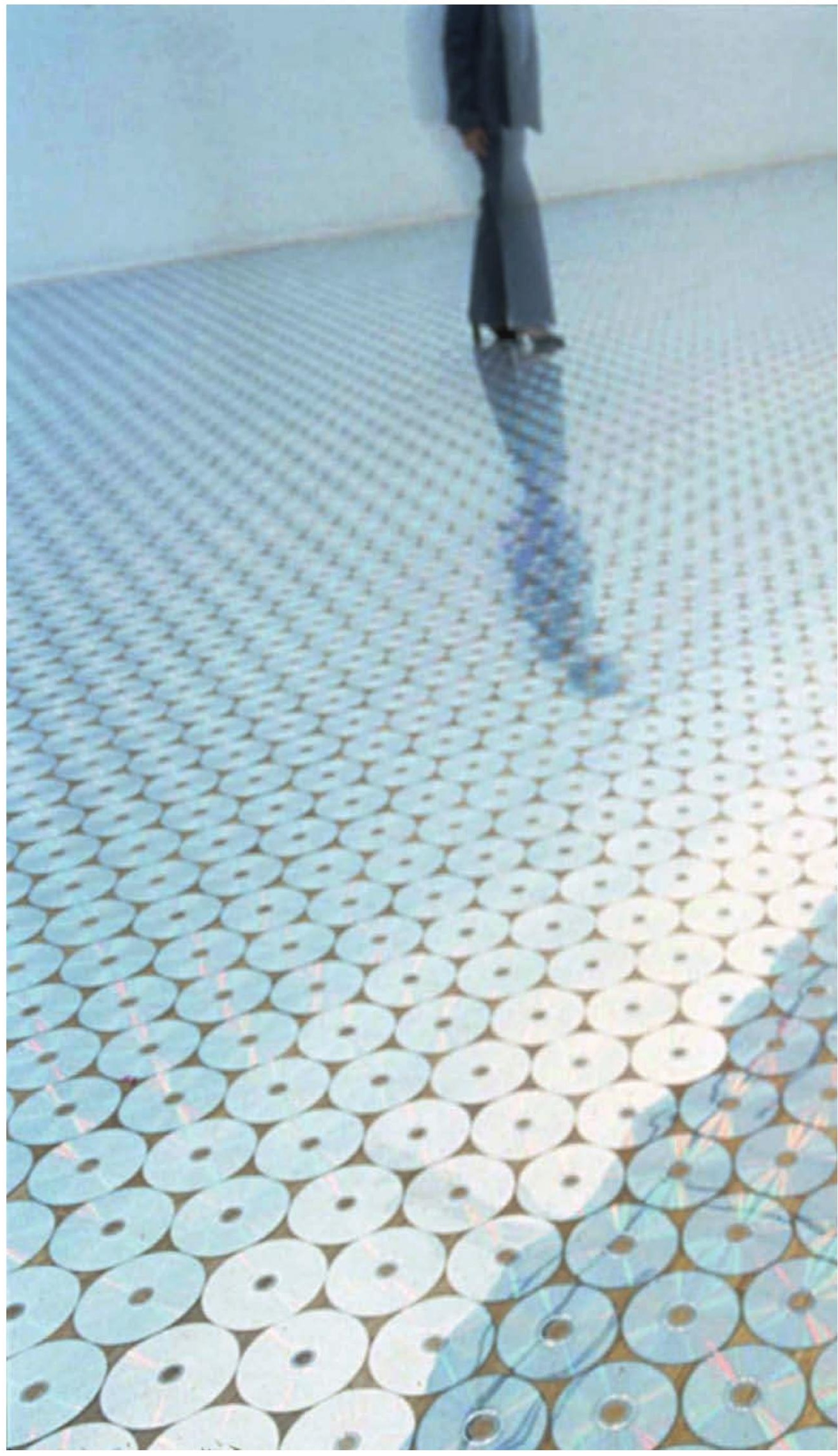




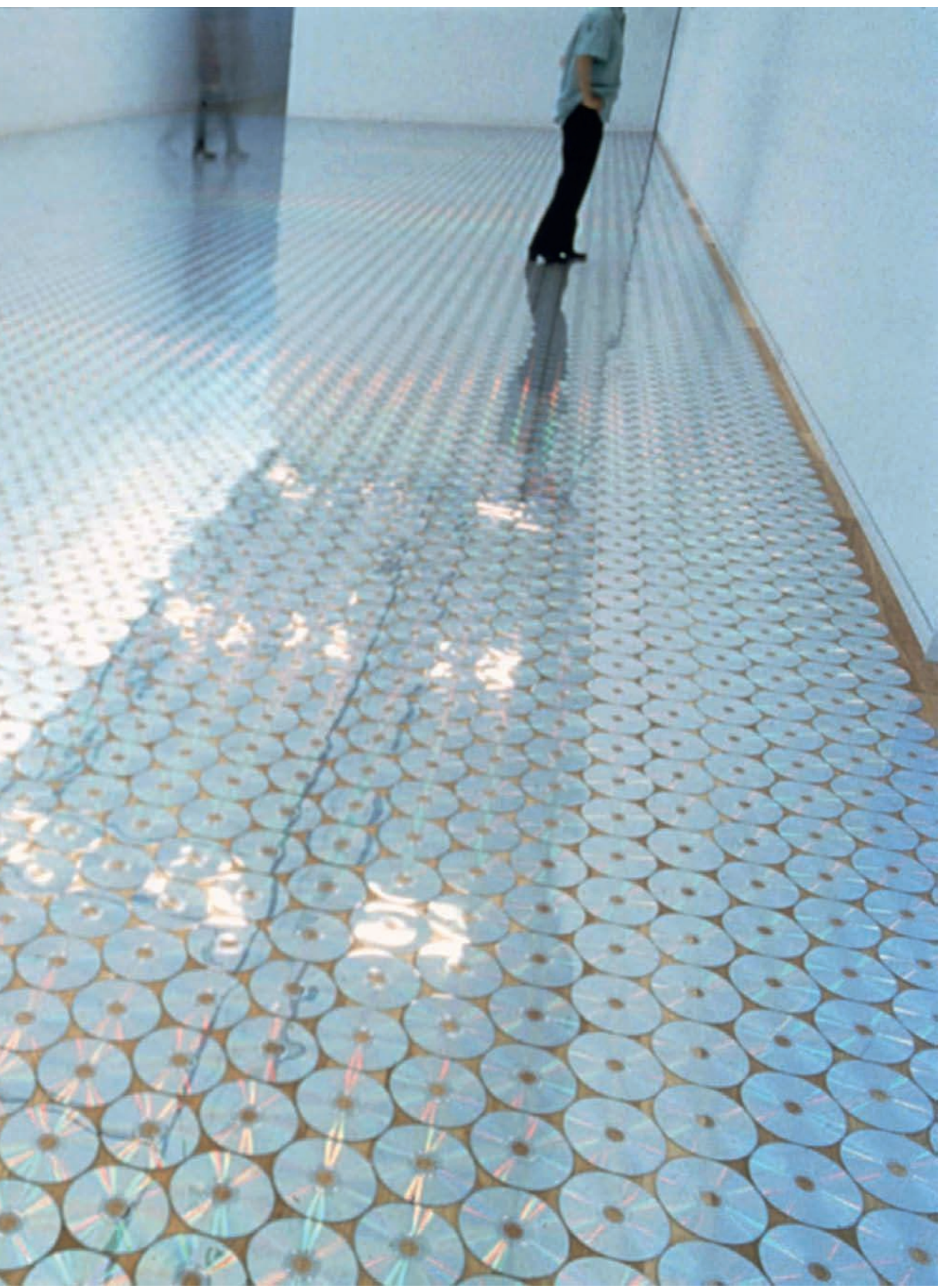


préconisée notamment par Gilbert Rouget (1968) - une des principales urgences étant selon lui de conserver des traces mémorielles de répertoires musicaux risquant de disparaître ou de se transformer radicalement à plus ou moins brève échéance en raison des mutations de leur environnement social.

S'il n'est pas nécessairement du ressort des ethnomusicologues de tenter de "sauvegarder" les musiques sur lesquelles ils travaillent, on peut cependant admettre avec Rouget qu'une telle démarche fait partie de la mission de ceux qui sont responsables d'archives musicales - et, qui plus est, comme c'est le cas des AIMP, d'archives rattachées à un musée, lieu de mémoire par excellence. On rappellera à ce propos la fameuse déclaration de Claude Lévi-Strauss, prononcée en 1967 à l'occasion de la sortie du deuxième volume des Mythologiques, Du miel et des cendres: "Ce n'est pas pour perpétuer cette diversité que je lutte, disaitil, mais pour en préserver le souvenir ${ }^{12}$.» Il est en effet évident, dans le cas qui nous occupe ici, que les bonnes intentions des uns et des autres ne suffiront pas à «sauvegarder » les cultures autochtones amazoniennes si les conditions matérielles de leur survie ne sont pas réunies. C'est ainsi qu'au moment d'écrire cet article nous apprenons que, suite à la découverte de gisements de pétrole, d'or et d'étain dans le sous-sol des terres ancestrales des Awajún et des Wampis, le gouvernement péruvien a décrété à leur égard des mesures d'expropriation radicales, suscitant des affrontements extrêmement violents entre les forces de l'ordre et les autochtones.

Aujourd'hui, alors que le CD consacré à la Musique des Awajún et des Wampis vient de paraître ${ }^{13}$, nous n'avons toujours pas reçu le contrat signé par les représentants de l'Odecofroc. On peut en effet concevoir que, vu la nature des événements auxquels les communautés du Cenepa sont actuellement confrontées, leurs urgences soient d'un autre ordre. Force nous est ainsi de constater que, face aux enjeux des décisions politiques, à la toute-puissance des intérêts économiques et à la brutalité de certaines méthodes policières, nos beaux soucis d'éthique au service de la diversité culturelle peuvent parfois paraître bien anecdotiques...

\section{Une berceuse pour la superstar du ghetto}

Durant la même période, soit début juin 2009, les AIMP ont été exposées à un autre cas problématique, qui s'est manifesté sous la forme d'un courriel envoyé à la maison de disques VDE-Gallo par une certaine Tracy Lynch-Sanchez, représentante de TLS Music Services, un bureau de consultants en production musicale établi à Burbank, Californie. L'auteur de ce message électronique nous demandait l'autorisation d'utiliser un échantillon (sample) d'une berceuse figurant dans un CD de notre collection publié en 1991, réalisé par l'ethnomusicologue italienne Serena Facci et intitulé : Zaïre. Entre les lacs et la forêt. La musique des Nande ${ }^{14}$. Après plus ample information, nous avons compris que l'échantillon en question était destiné à être intégré, sous forme d'une boucle (loop) musicale, à l'introduction du morceau Need to find $^{15}$ de la chanteuse jamaïcaine Terry Lynn, associée au DJ et producteur anglo-suédois Johan Hugo. Curieusement, selon le courriel de Lynch-Sanchez, l'usage de cette berceuse congolaise dans un tel contexte était censé appuyer l'identité afro-jamaïcaine de l'artiste.

Désireux de savoir de quoi il retournait, nous avons découvert que Lynn, étoile montante du dub jamaïcain, avait récemment été proclamée « Ghetto Superstar " 


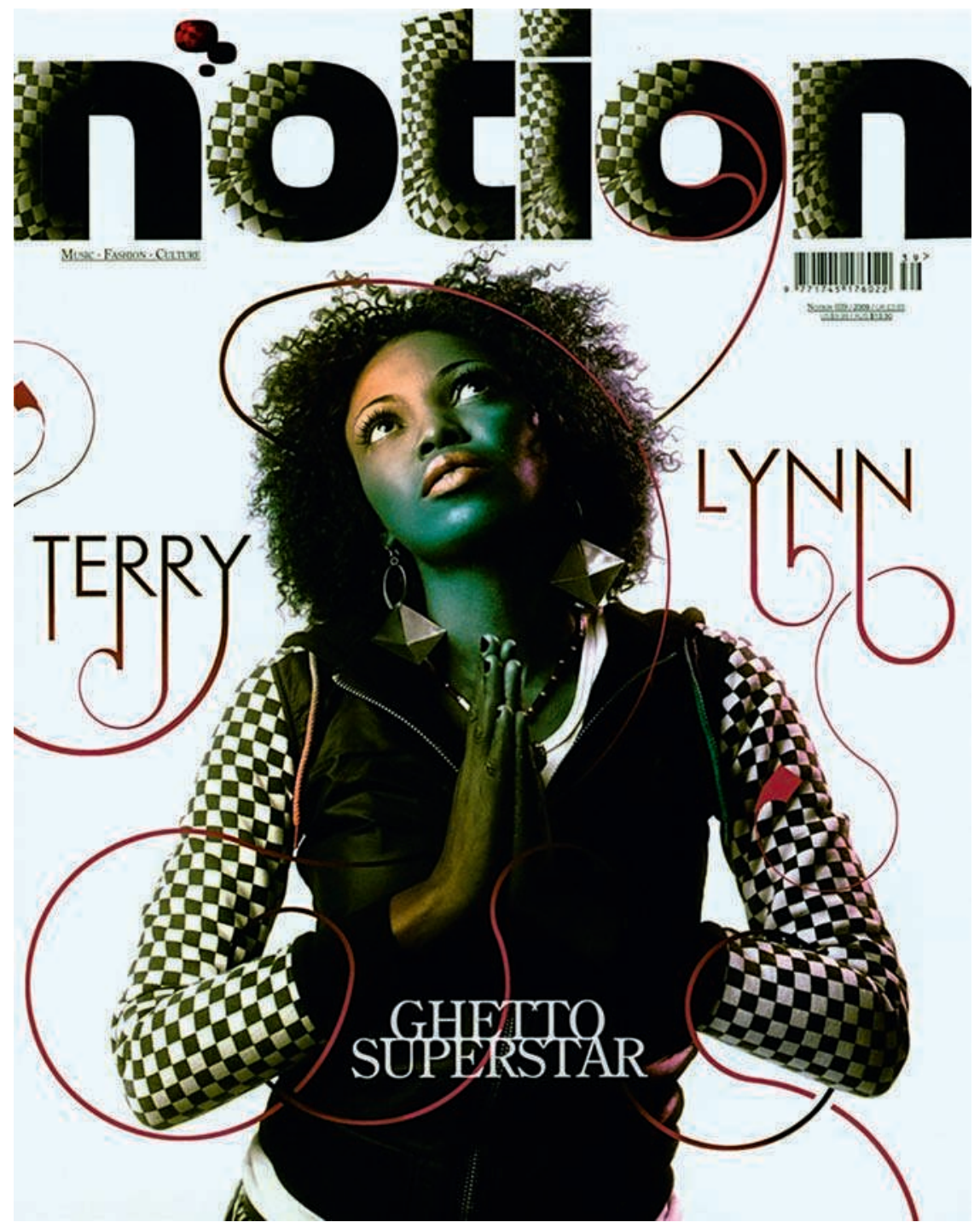

Fig. 5 Couverture du magazine Notion avec Terry Lynn, Notion UK n 9, 2009. Photo Mark Cant. Droits réservés.

par le magazine online Notion UK, dont elle avait fait la couverture de l'édition du 5 mai $2009^{16}$. Dans l'article très lyrique qui lui était consacré dans ces pages, on apprenait notamment qu'elle était la dernière d'une famille de neuf enfants nés dans un quartier pauvre de Kingston et qu'elle serait la «voix d'une génération désespérée. Héritière d'une histoire cruelle, guerrière de la paix dans un lieu de dépravation, de crimes violents et d'effondrement politique. Son artfait partie d'un mouvement passionné pour le changement en Jamaïque, à la fois écho des appels de ses prédécesseurs et incitation à un sens de l'urgence puissant et revigoré [...] ".

Selon le site de TLS Music, la chanson Need to find faisait partie d'un certain "Red Stripe Website Project", dont nous avons découvert qu'il s'agissait d'une opération de marketing lancée par la marque de bière jamaïcaine Red Stripe, sponsor du projet, dans l'intention de célébrer «l'influence mondiale, l'importance et l'attrait des rythmes et de la pulsation de la musique jamaïcaine ${ }^{17}$ ».
Les extraits musicaux de

la berceuse nande et de sa reprise par Terry Lynn et Johan Hugo sont consultables sur http://gradhiva.revues.org/1813

\footnotetext{
-

16. http://kingstonlogic.com/index. php?id=17?.

17. « The worldwide influence, importance and appeal of the rhythms and pulse of Jamaican music », http://gigulate. com/story/New-Terry-Lynn--amp-Johan-Hugo----Need-To-Find.
} 
-

18. Les Banande, ou Nande - ethnie à laquelle appartenait la chanteuse Marguerite, interprète des deux berceuses en 1988 -, vivent dans la province du Nord-Kivu, dans la République démocratique du Congo (exZaïre). Depuis 1997, le Kivu est l'objet d'affrontements meurtriers quasi incessants entre l'armée congolaise et l'opposition menée par Laurent Nkunda. Avec les Hutu, les Tutsi et les Banyarwanda, les Banande sont parmi les populations les plus touchées par ces conflits, qui ont fait d'innombrables victimes.
L'affaire nous paraissant plutôt suspecte, nous avons donc décidé dans un premier temps de nous opposer formellement à tout usage gracieux de l'extrait de «notre» berceuse dans ce cadre. La réponse nous est parvenue par retour de courrier, nous informant que la chanson en question, dont on nous disait qu'elle "ne sera pas distribuée commercialement», allait pourtant être diffusée - du moins sur Internet - avec une première perspective de cinquante mille unités, pour lesquelles le producteur nous proposait une avance de 3000 dollars... C'était à n'y rien comprendre! Étions-nous exposés à un nouveau "cas Deep Forest ", ou au contraire à une situation où la production d'une artiste était pour une fois soucieuse de partager ses droits d'auteur de façon solidaire avec les interprètes dont elle entendait utiliser la musique?

Ne souhaitant pas prendre la décision seuls, nous avons contacté Serena Facci afin de connaître son point de vue. «Comme tu le sais, m'a-t-elle répondu, le territoire des Banande ${ }^{18}$ est en guerre. Je vais chercher à contacter l'obstétricienne italienne qui m'avait mise en contact avec les femmes de Lukanga pour savoir s'il est possible de donner l'argent à la maternité de Lukanga ou à quelque autre œuvre caritative sur place. J'ai confiance en cette femme, et je ne vois pas d'autre solution pour le moment [...].»

Dans un courrier ultérieur, Serena nous apprit que Marguerite, la sage-femme qui avait interprété les deux berceuses, était entre-temps décédée, mais que l'obstétricienne italienne poursuivait son travail dans un Kivu toujours ravagé par la guerre. Estimant que la somme proposée "pourra être utilisée au mieux, à la mémoire de Marguerite, qui était vraiment une vieille femme " formidable, l'ethnomusicologue italienne nous demanda aussi de transmettre le message suivant à TLS Music: The Congolese lullaby in Need to find was sung in 1988 by Marguerite, a Munande woman, in a village near Butembo (Democratic Republic of Congo). «Il faut être clair avec les musiciens, ajoutait-elle. Ils peuvent utiliser cette brève note ou pas, mais ils ne doivent absolument pas inventer autre chose ! »

Après réflexion, nous avons finalement décidé d'un commun accord d'accepter la proposition de TLS Music, jugeant sa démarche relativement honnête et transparente, et en tout cas assez rare pour mériter d'être saluée. En outre, nous étions convaincus que la chanson allait de toute façon se retrouver sur le marché - le master en était achevé depuis plusieurs semaines et quelques sites la diffusaient déjà au moment où nous devions prendre notre décision. Une action en justice aurait en outre risqué d'être interminable et son issue plus qu'incertaine. Par ailleurs, même une fois les frais déduits, le montant proposé était suffisamment important pour qu'il puisse avoir un effet significatif sur la situation financière du dispensaire du Nord-Kivu.

La chanson Need to find a été mise en ligne le 8 juillet 2009; mais, bien que les accords aient été signés, l'argent n'est toujours pas arrivé...

\section{Épilogue}

Ces deux exemples n'ont certes pas permis d'aborder toutes les questions relatives au copyright dans le cas de productions musicales résultant d'enregistrements de terrain. Mais ils ont au moins eu le mérite de souligner quelques points essentiels en ce qui concerne la déontologie professionnelle et les obligations qu'elle implique pour l'ethnomusicologue (qui étudie une musique et en diffuse la connaissance), le preneur de son (souvent la même personne, qui en saisit les 


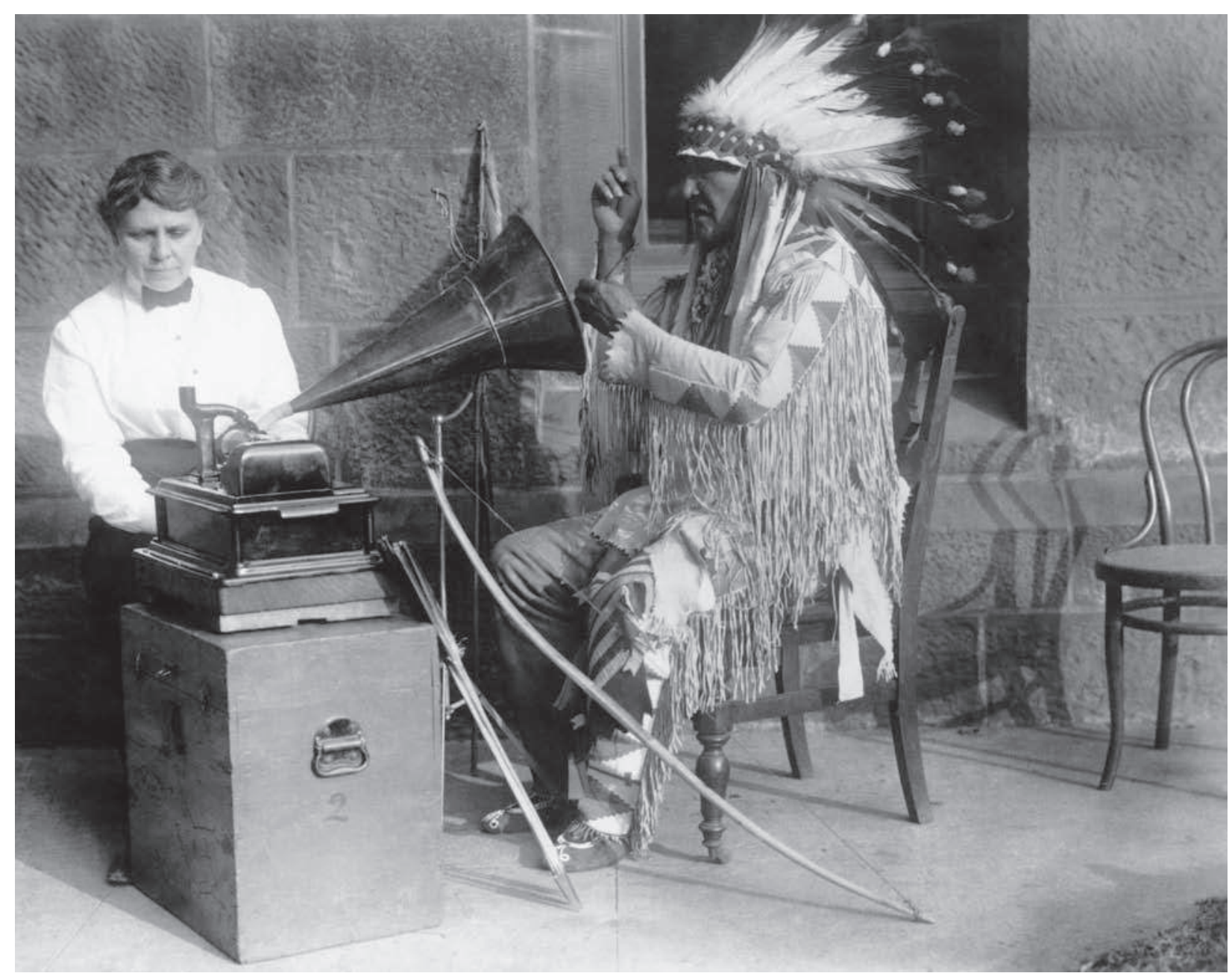

Fig. 7 L'ethnologue Frances Densmore effectuant un enregistrement de musiques d'Indiens d'Amérique, 1895 ๑ Photo MPI/Getty Images.

traces sonores), l'archiviste (qui conserve et classe ces traces) et l'éditeur musical (qui en propose un témoignage sous forme d'une publication aussi bien documentée que possible).

Chacun à sa manière, les deux cas étudiés dans ces pages démontrent que ces activités peuvent être porteuses d'enjeux et génératrices de responsabilités : sauvegarder des traces mémorielles d'un patrimoine menacé pour le premier et contribuer à défendre les intérêts de ses détenteurs pour le second. Tous deux sont ainsi concernés par les deux catégories juridiques établies par l'OMPI : celle des droits patrimoniaux et celle du droit moral. On notera que, dans le texte de l'OMPI cité plus haut, l'ayant droit est désigné indifféremment par les termes d'«auteur» et de "créateur». Or, nous l'avons vu, ces notions ne sont pas pertinentes dans notre cas amazonien; en revanche, la qualité de «dépositaire " a été mentionnée : «l'individu détenteur d'un chant n'en est pas pour autant le propriétaire; il n'en est que le dépositaire, dans la mesure où il l'a acquis de façon régulière, et ce dépôt n'est en tout cas ni collectif, ni négociable, ni transmissible hors de ce cadre " (supra). L'Odecofroc n'est à cet égard que le représentant délégué par la collectivité des individus awajún et wampis dépositaires des chants. 
-

19. Marc Perlman, professeur associé de musique à la Brown University de Providence (Rhode Island, États-Unis], communication orale, 6 juillet 2009.

20. http://portal.unesco.org/fr/ ev.php-URL ID=12025\&URL_DO=DO TOPIC\&URL_SECTION=-471.html.

21. http://www.wipo.int/treaties/fr/ ip/berne/trtdocs wo001.html.
Qui sont en revanche les ayants droit sur l'enregistrement de notre berceuse congolaise, d'auteur évidemment anonyme et interprétée il y a plus de vingt ans par une villageoise du Kivu entre-temps décédée? Et, plus précisément, qui est ici autorisé à décider des questions relatives au droit moral afférant à l'usage de ce phonogramme par des tiers? Ces questions sont évidemment d'autant plus difficiles à trancher qu'elles font appel à une appréciation subjective de l'opportunité survenue, mais dont les retombées sont potentiellement très concrètes. Nous pouvons assumer que cette mélopée ressortit bien au domaine de la "propriété intellectuelle collective" des Banande, auquel des droits imprescriptibles sont attachés, y compris dans le cas qui nous concerne. Mais, en tout état de cause, selon les dispositions prises lors de sa publication, le droit moral sur le phonogramme est effectivement du ressort partagé de l'«auteur " (l'ethnomusicologue Serena Facci) et des deux entités "productrices " que constituent les AIMP et la maison de disques VDE-Gallo, toutes deux engagées moralement par leur représentant.

Il faut cependant signaler, comme me le faisait récemment remarquer l'ethnomusicologue Marc Perlman, spécialisé notamment dans les questions juridiques $^{19}$, que le gouvernement de la République démocratique du Congo serait théoriquement en droit de réclamer l'intégralité des royalties perçues sur cette berceuse. En effet, la République démocratique du Congo n'a à ce jour ratifié ni la Convention pour la sauvegarde du patrimoine culturel immatériel (2003) de l'Unesco, ni celle sur la protection et la promotion de la diversité des expressions culturelles ${ }^{20}$ (2005). En revanche, comme le Pérou d'ailleurs, elle est signataire de la Convention de Berne pour la protection des œuvres littéraires et artistiques, ce qui sous-entend que sa législation est "conforme aux normes internationales". Or, si l'article 12 de cette convention stipule que «les auteurs d'œuvres littéraires ou artistiques jouissent du droit exclusif d'autoriser les adaptations, arrangements et autres transformations de leurs œuvres ${ }^{21}$ ", l'article 19 dit bien que «les dispositions de la présente convention n'empêchent pas de revendiquer l'application de dispositions plus larges qui seraient édictées par la législation d'un pays de l'Union ", en l'occurrence celle de l'État congolais.

Mais il était pour nous évident que cette éventualité n'était pas à considérer. Et à cet égard je ne peux que souscrire, ici encore, aux propos de Seeger lorsqu'il soutient que «les questions qui touchent à la propriété intellectuelle [...] sont trop importantes pour être laissées aux seuls juristes, car elles relèvent non seulement du droit (ce qu'on peut faire), mais aussi de l'éthique (ce qu'on devrait faire)»(2007:70-71).

Musée d'ethnographie de Genève Directeur des Ateliers d'ethnomusicologie de Genève la.adem@mail-box.ch

mots clés / keywords : propriété intellectuelle // intellectual property • droits d'auteur // copyright • échantillonnage // sampling • ethnomusicologie d'urgence // salvage ethnomusicology • déontologie // deontology. 


\section{Bibliographie}

\author{
AuberT, Laurent \\ 2001 La Musique de l'autre. Les nouveaux défis \\ de l'ethnomusicologie. Genève, Georg éditeur. \\ 2010 « Du "bon usage" des musiques du \\ monde. Questions sur une éthique de la \\ diversité culturelle », in Trottier (dir.) 2010. \\ Aubert, Laurent (dir.) \\ 2009 Mémoire vive. Hommages à Constantin \\ Brailoiu. Genève-Gollion, MEG-Infolio \\ [«Tabou 6 》).

\section{DASEN, Patrik Vincent} \\ 2009 « Histoire d'une collecte. "Nagkamsa \\ Awajún nampet dakubau atatui Cenepia" », \\ in Aubert (dir.) 2009: 257-264.

\section{Duvignaud, Jean et KHAzNADAR,} \\ Chérif (dir.) \\ 2004 Le Patrimoine culturel immatériel. \\ Les enjeux, les problématiques, les pratiques. \\ Arles, Babel [《Internationale de l'imaginaire 17 »].

\section{FAccI, Serena} \\ 1991 Zaïre. Entre les lacs et la forêt. La \\ musique des Nande, CD AIMP XXIII/VDE-652.
}

\section{FELD, Steven}

1996 « Pygmy POP. A Genealogy of

Schizophonic Mimesis », Yearbook for

Traditional Music 28 : 1-35.

HuXley, Francis et NARBY, Jeremy

2002 Chamanes au fil du temps. Cinq cents ans sur la piste du savoir. Paris, Albin Michel.

\section{NARBY, Jeremy}

2005 Intelligence dans la nature. En quête du savoir. Paris, Buchet-Chastel.

PENNeWAert, Eddy (dir.)

2007 Le Patrimoine culturel immatériel.

Droits des peuples et droits d'auteur.

Bruxelles, Colophon Éditions.

\section{Riol, Raúl et NARBY, Jeremy}

2009 Pérou. Musique des Awajún et des

Wampis. Amazonie, vallée du Cenepa,

CD AIMP XCII/NDE-1279.

\section{Rouget, Gilbert}

1968 «L'ethnomusicologie », in Jean Poirier

[éd.], Ethnologie générale. Paris, Gallimard

[«Encyclopédie de la Pléiade 24 ») : 1339-1390.

\author{
SeEgER, Anthony \\ 2007 « La propriété intellectuelle, les archives \\ et les collections audiovisuelles », \\ in Pennewaert (dir.) 2007 : 69-89. \\ Seeger, Anthony et Chaudhuri, \\ Shubha (éd.) \\ 2004 Archives for the Future. Global \\ Perspectives on Audiovisual Archives in \\ the 21st Century. Calcutta, Seagull Books. \\ TROTTIER, Danick (dir.) \\ 2010 «Éthique, droit et musique », \\ Cahiers de la Société québécoise de recherche \\ en musique 11(1-2). \\ ZEMP, Hugo \\ 1996 «The/An Ethnomusicologist and the \\ Record Business », Yearbook for Traditional \\ Music 28 : 36-55.
}

\section{Résumé / Abstract}

Laurent Aubert, Woodstock en Amazonie et la superstar du ghetto de Kingston. Les droits patrimoniaux et le droit moral face aux réalités du terrain - Chaque CD publié dans la collection AIMP (Archives internationales de musique populaire) fait, comme il se doit, l'objet d'un «contrat de cession et d'édition d'enregistrements musicaux », établi soit avec «l'artiste » (l'interprète ou son représentant légal), soit, plus fréquemment, avec «l'auteur», c'est-à-dire la personne (ethnomusicologue, anthropologue ou preneur de son) ayant réalisé les enregistrements. Dans ce dernier cas, le contrat stipule que l'auteur garantit être en possession des droits de cession des enregistrements concernés. Ces dispositions peuvent cependant poser certains problèmes, ainsi que l'a récemment démontré la publication d'un CD sur la musique d'une communauté amérindienne d'Amazonie péruvienne, chez qui des notions comme le droit d'auteur ou la propriété intellectuelle ne correspondent à aucune réalité. La question des droits voisins se pose aussi parfois, comme ce fut le cas avec une berceuse congolaise dont un échantillon (sample) a été intégré à une chanson d'une interprète de dub jamaïcain. De tels exemples montrent que le droit et la déontologie peuvent s'affronter dans la solution de problèmes impliquant des considérations à la fois patrimoniales, artistiques, juridiques et économiques.
Laurent Aubert, Woodstock in Amazonia and the Kingston Ghetto Superstar. Patrimonial and moral rights confronted with reality in the field - Every CD released by the IAPM collection [International archives of popular music] is necessarily the object of a "musical recordings copyright assignment agreement" signed either with the "artist" (the performer or his legal representative) or, more commonly, with the "author"-i.e. the person who recorded the music (e.g. ethnomusicologists, anthropologists or sound man). In the latter case, contracts stipulate that the author must copyright for the recordings.

These stipulations, however, are not without posing certain problems, as witnessed by the recent release of a $C D$ of music from an Amerindian community in the Peruvian Amazon, for whom such notions as copyright and intellectual property are devoid of significance. Related rights may also be a problem, as in the case of a Congolese lullaby sampled into a Jamaican dub track. Such examples demonstrate that law and deontology may come into conflict in the resolution of problems involving artistic, patrimonial, legal and economic concerns. 